\title{
Supramaximal flow in asthmatic patients
}

\author{
H. Sala*, A. Fernández*, S. Guardia*, A. González*, D. Rodenstein\#
}

\begin{abstract}
Supramaximal flow in asthmatic patients. H. Sala, A. Fernández, S. Guardia, A. González, D. Rodenstein. (C) ERS Journals Ltd 2002.

ABSTRACT: In normal subjects supramaximal flows (SF) are known to be correlated with flow limitation. To further understand the mechanisms involved in SF this correlation and the influence of salbutamol and methacholine administration on SF have been investigated in asthmatic subjects.

Protocol A involved obtaining basal maximal expiratory flow/volume curves and interrupted curves through a fast valve from 36 asthmatic patients. Maximal flow at $50 \%$ of forced vital capacity $\left(V^{\prime} \max _{50}\right)$ increase $\left(\Delta V^{\prime} \max _{50}\right)$ was compared between basal curves and envelope curves passing through SF peaks obtained after each interruption.

Protocol B involved the study of 33 asthmatic patients after salbutamol administration and 12 asthmatic patients after methacholine challenge. $\Delta V^{\prime} \max _{50}$ between basal versus interrupted curves were analysed $\left(\Delta V^{\prime} \max _{50} \mathrm{~B}\right)$. Similar procedures were performed after salbutamol $\left(\Delta V^{\prime} \max _{50} S\right)$ and after methacholine administration $\left(\Delta V^{\prime} \max _{50} \mathrm{M}\right)$. A significant negative correlation between forced expiratory volume in one second and $\Delta V^{\prime} \max _{50}$ was observed. SF decreased significantly after salbutamol administration and increased significantly after methacholine.

The results of this study suggest that in asthmatic patients participation of "pendelluft" in SF increases as airflow limitation increases.
\end{abstract} Eur Respir J 2002; 19: 1003-1007.

*Posadas Hospital, El Palomar Buenos Aires, Argentina. "Pneumonology Unit, Saint Luc Hospital, Catholic University of Louvain, Brussels, Begium.

Correspondence: H. Sala

Gurruchaga 1831

PC1414-Capital Federal

Argentina

Fax: 541146547892

E-mail:drsala@tutopia.com

Keywords: Asthma

lung inhomogeneity pendelluft

supramaximal flow

Received: October 152001

Accepted after revision January 10 2002
When a partial maximal expiratory flow/volume (MEFV) curve is performed by starting the manoeuvre at a volume below total lung capacity (TLC), a transient peak flow is obtained, exceeding the flow observed in the MEFV curve for the same lung volume. This flow is referred to as supramaximal flow (SF), which is also observed during coughing [1]. Several mechanisms have been proposed to explain SF $[2,3]$. Working with interrupted curves, the authors have observed that the longer the occlusion valve time, the higher the SF. The authors have also observed that SF correlates with flow limitation and therefore proposed that refilling the fast units by air from the slow units during interruptions, a mechanism called "pendelluft", is also a main contributing factor to SF [4].

In order to understand further the underlying mechanisms implied in SF, the SF behaviour in asthmatic subjects, and the correlation between SF and airflow limitation indices and the SF response when changes in lung emptying heterogeneity were performed were investigated.

\section{Materials and methods}

Two protocols were performed, both included patients meeting both clinical and spirometric asthma criteria. Written consent was obtained from all patients and the author's hospital ethics committee approved both protocols.

Protocol A included 36 patients. The anthropometric and basal spirometric data from these patients are depicted in table 1.

Baseline MEFV curves were carried out according to American Thoracic Society (ATS) criteria [5]. Thereafter, patients repeated manoeuvres exhaling from TLC to residual volume through a specially devised fast valve, which interrupted flow 6 times $\cdot \mathrm{s}^{-1}$ with an open:close ratio of 4:1. After each valve interruption a transient SF was observed. An envelope curve passing through SF peaks was depicted, and an increase in flow $50 \%$ of the basal forced vital capacity (FVC) was measured between basal curve and the envelope curve $\left(\Delta V^{\prime} \max _{50}\right)$ (fig. 1).

Interrupted curves were accepted if agreement in FVC and forced expiratory volume in one second (FEV1) between at least three of them was $<5 \%$ and $<200 \mathrm{~mL}$ and the FVC agreement with basal

Table 1.-Antropometric and lung-function data of the 36 patients in Protocol A

\begin{tabular}{lr}
\hline Age & $45 \pm 16$ \\
Height cm & $161 \pm 10$ \\
Weight kg & $70 \pm 18$ \\
FVC $\%$ & $86 \pm 16$ \\
FEV $\%$ & $60 \pm 20$ \\
\hline
\end{tabular}

Data are presented as mean \pm SD. FVC: forced vital capacity; FEV1: forced expiratory volume in one second. 


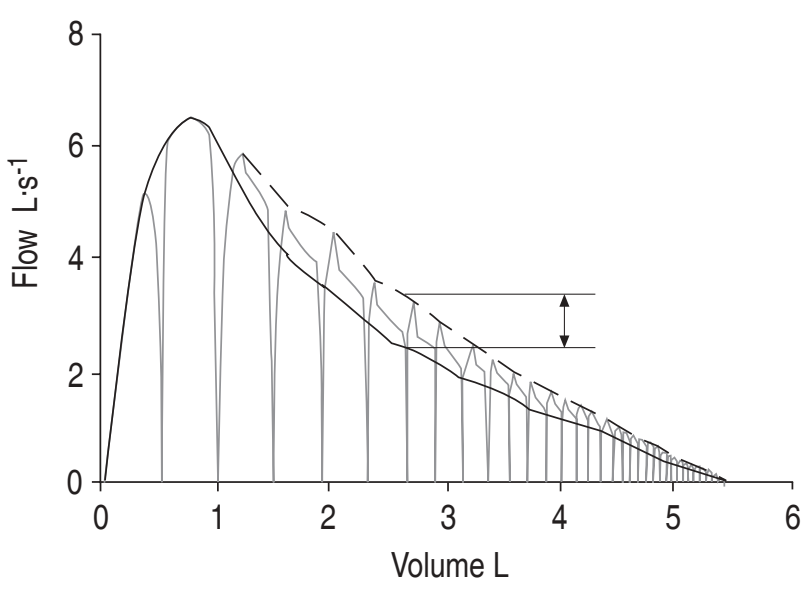

Fig. 1.-Basal (-) and $6 \mathrm{~Hz}(--$-) interrupted maximal expiratory flow/volume curve. An envelope curve (…….) passing through the peaks of supramaximal flows is depicted. Arrows represent maximal flow at $50 \%$ of forced vital capacity, which was determined by matching the curves at total lung capacity.

MEFV curves was $<5 \%$. Comparison between FEV1 (in absolute value) obtained from interrupted curves and FEV1 obtained from basal curves was performed $(\triangle \mathrm{FEV} 1)$ by using paired t-tests. Correlation between $\Delta V^{\prime} \max _{50}$ and basal FEV1 $\%$ pred value was performed by the Pearson correlation test.

Protocol B included 45 asthmatic patients. Anthropometric and basal spirometric data of the patients included in this protocol are depicted in table 2 .

The asthmatic patients included in this protocol performed basal and interrupted curves following the same methodology as in Protocol A. Two metered-dose inhaler salbutamol puffs $(200 \mu \mathrm{g})$ were administered to 33 out of 36 patients included in protocol A (group S). Presalbutamol interrupted and basal curves were carried out in the same way as in the previous manoeuvres. An increase in flow between the basal MEFV curves and basal envelope curve at $50 \%$ of basal FVC was measured $\left(\Delta V^{\prime} \max _{50} \mathrm{~B}\right)$. Increase in flow between postsalbutamol basal and interrupted curves at $50 \%$ of FVC were also measured $\left(\Delta V^{\prime} \max _{50} \mathrm{~S}\right)$. Comparison between $\Delta V^{\prime} \max _{50} \mathrm{~S}$ and $\Delta V^{\prime} \max _{50} \mathrm{~B}$ was performed $\left(\Delta V^{\prime} \max _{50} \mathrm{~S} / \mathrm{B}\right)$ by paired t-test.

Table 2. - Anthropometric and lung-function data of the 45 patients in Protocol B

Salbutamol Methacholine p-value

\begin{tabular}{lccl}
\hline Subjects n & 33 & 12 & \\
Age yrs & $44 \pm 16$ & $47 \pm 13$ & $>0.05$ \\
Height cm & $161 \pm 10$ & $163 \pm 10$ & $>0.05$ \\
Weight kg & $70 \pm 18$ & $73 \pm 22$ & $>0.05$ \\
Basal FEV $\%$ pred & $59 \pm 18$ & $61 \pm 25$ & $>0.05$ \\
Basal $\Delta V^{\prime} \max _{50} \%$ & $91 \pm 60$ & $58 \pm 22$ & \\
FEV1 S/M \% pred & $71 \pm 18$ & $44 \pm 18$ & \\
\hline
\end{tabular}

Data are presented as mean \pm SD unless otherwise stated. FEV1: forced expiratory volume in one second; $\Delta V^{\prime} \max _{50}$ : change in maximal flow at $50 \%$ forced vital capacity; S: salbutamol; M: methacholine.
In 12 new asthmatic patients (group M) methacholine bronchoprovocation test was carried out using increasing concentrations of buffered chloridrate of methacholine $\left(1,2,5,10,25,100 \mathrm{mg} \cdot \mathrm{mL}^{-1}\right)$ until a fall $>20 \%$ in FEV1 was observed [6]. The last noninterrupted curve was selected to compare it with the interrupted curve performed immediately after. Increase in flow between the basal MEFV curve and the interrupted envelope curve at $50 \%$ basal FVC was measured $\left(\Delta V^{\prime} \max _{50} \mathrm{~B}\right)$. Increase in flow between the noninterrupted postmethacholine curve and the interrupted postmethacholine curve at 50\% FVC, obtained from the noninterrupted curve, was also measured $\left(\Delta V^{\prime} \max _{50} \mathrm{M}\right)$. A paired t-test was used to compare $\Delta V^{\prime} \max _{50} \mathrm{M}$ and $\Delta V^{\prime} \max _{50} \mathrm{~B} \quad\left(\Delta V^{\prime} \max _{50} \mathrm{M} / \mathrm{B}\right)$. The Pearson correlation test was applied to analyse the correlation between $\Delta V^{\prime} \max _{50}$ and the change in $\triangle F E V 1$ induced by both methacholine and salbutamol administration.

All manoeuvres were performed using a rolling seal spirometer (Sensormedics, USA), with a flat frequency response up to $7 \mathrm{~Hz}$ (manufacturer's unpublished data) with a resistance of $<2 \mathrm{cmH} \mathrm{H}_{2} \mathrm{O} \cdot \mathrm{L}^{-1} \cdot \mathrm{s}^{-1}$ at a flow of $12 \mathrm{~L} \cdot \mathrm{s}^{-1}$. The spirometer was coupled to a personal computer. The equipment complied with current ATS recommendations [7]. The inertial characteristics of the spirometer were tested at 3 and $6 \mathrm{~Hz}$ using an explosive decompressor (ATSOK, Argentina) [8] (fig. 2).
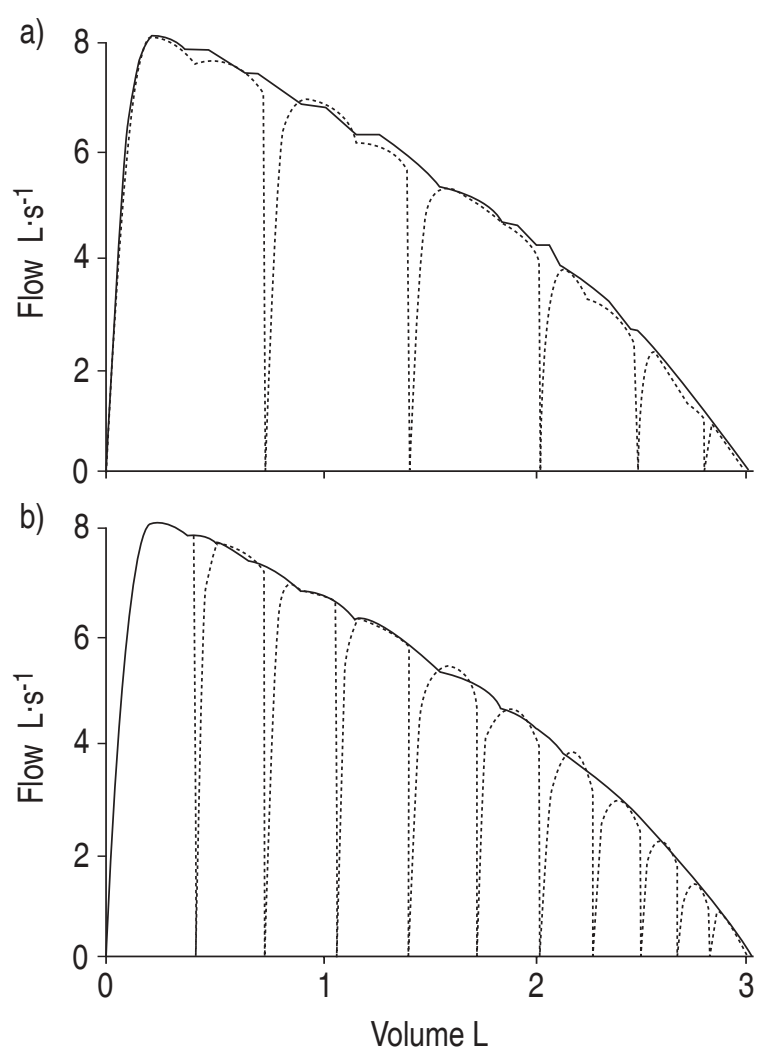

Fig. 2.-Basal (-) and a) $3 \mathrm{~Hz}(---)$ and b) $6 \mathrm{~Hz}(---)$ interrupted maximal expiratory flow/volume curves generated by an explosive decompressor. 


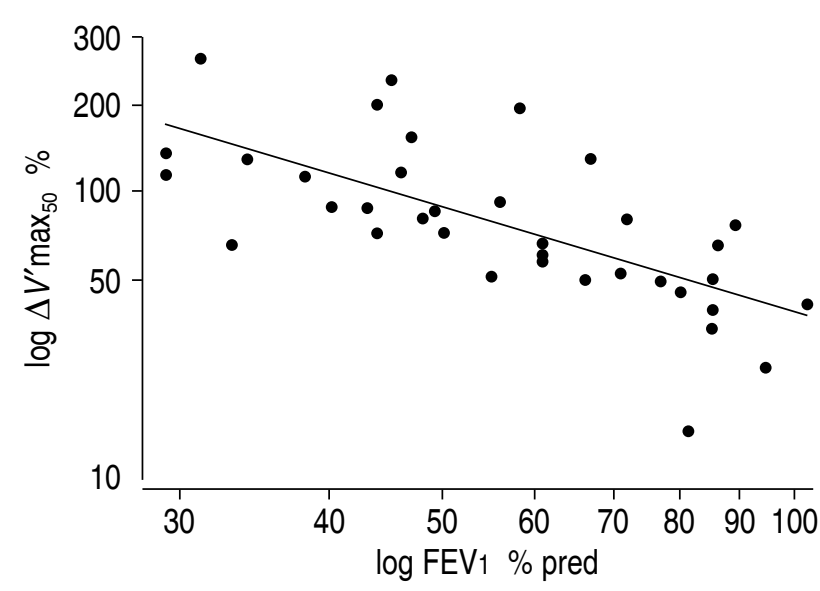

Fig. 3.-Correlation between forced expiratory volume in one second $\left(\mathrm{FEV}_{1}\right)$ and maximal flow at $50 \%$ of forced vital capacity $\left(V^{\prime} \max _{50}\right) . r=-0.683$.

\section{Results}

\section{Protocol $A$}

There was a significant negative correlation between baseline $\mathrm{FEV} 1 \%$ pred value and $\Delta V^{\prime} \max _{50}$, so that the lower the FEV1, the higher the $\Delta V^{\prime} \max _{50}(\mathrm{r}=-0.683)$ (fig. 3). FEV1 obtained from interrupted curves was significantly higher than the basal FEV1 $(1.8 \pm 0.64 \mathrm{~L}$ versus $1.74 \pm 0.63 \mathrm{~L})(\mathrm{p}=0.01226)$.

\section{Protocol B}

Salbutamol resulted in an increase in FEV1 by $21.4 \%$ in comparison to presalbutamol values $(\mathrm{p}=$ $0.00748)$. In addition, during interrupted exhalations, $\Delta V^{\prime} \max _{50}$ decreased after salbutamol in comparison to basal $\Delta V^{\prime} \max _{50} \quad(90.75 \pm 60 \%$ versus $54.15 \pm 24 \%)$ $(\mathrm{p}<0.01)$ (fig. 4).

Methacholine challenge led to a $29.4 \%$ decrease in FEV1 $(\mathrm{p}=0.000077)$. Postmethacholine $\Delta V^{\prime} \max _{50}$ increased in comparison to the premethacholine value $(57.66 \pm 22 \%$ versus $124.41 \pm 48 \%)(\mathrm{p}<0.02)$ (fig. 5$)$.

Analyses of the interrupted curves in the asthmatic patients of Protocol A produced two different forms

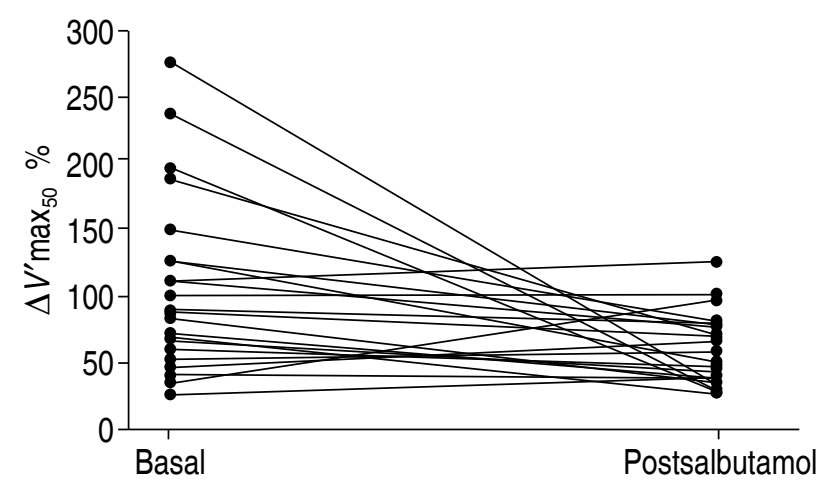

Fig. 4.-Comparison of basal and postsalbutamol maximal flow at $50 \%$ of forced vital capacity $\left(V^{\prime} \max _{50}\right)$. Data are presented as per cent increase. $\mathrm{p}<0.01$.

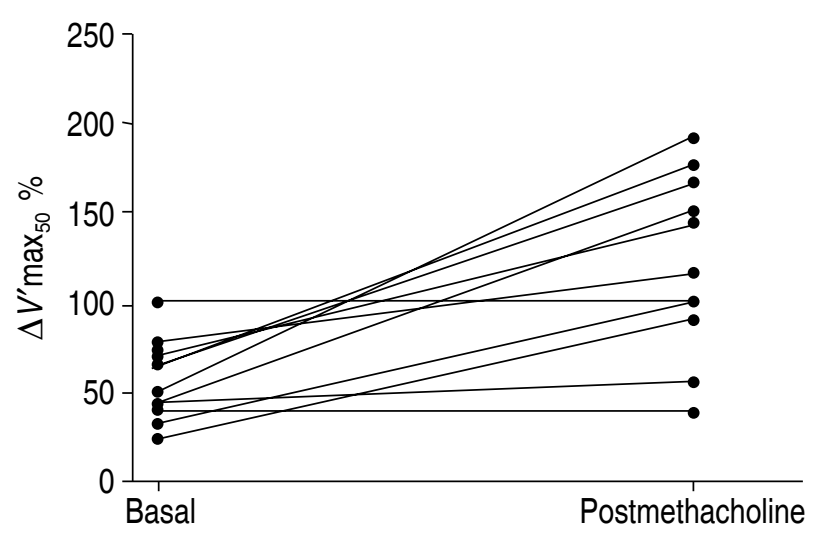

Fig. 5.-Comparison of maximal flow at 50\% of forced vital capacity $\left(V^{\prime} \max _{50}\right)$ between basal and postmethacholine administration. Data are presented as per cent increase. $\mathrm{p}<0.02$.

of curves. In form "a" (fig. 6) the flow immediately after the SF peak returned to the basal curve value. In form "b" (fig. 7) the flow immediately after the SF peak remained higher than the flow observed in the basal curve for the same lung volume, until the next interruption was performed. To further analyse the forms of the interrupted curves, the patients were allocated into two subgroups. Subgroup 1 included 24 patients in which FEV1 increased when the interrupted curves were performed in comparison with basal FEV1. Subgroup 2 included 12 patients in which basal FEV1 of the noninterrupted curves was higher than FEV1 obtained from interrupted curves. Comparison of both forms according to FEV1 behaviour is depicted in figure 8 . Prevalence of form "b" was significantly higher in subgroup 1 patients $(p=0.00003)$.

\section{Discussion}

Before discussing the results obtained from this study some methodological considerations must be taken into account. Despite a lower frequency response and higher inertia in comparison with a

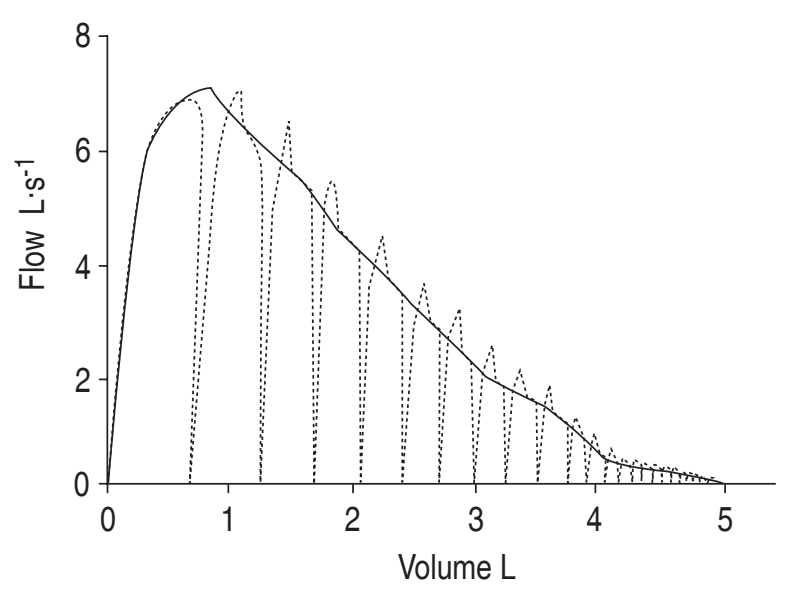

Fig. 6.-Form a curve. The flow after supramaximal flow returns to basal curve. 


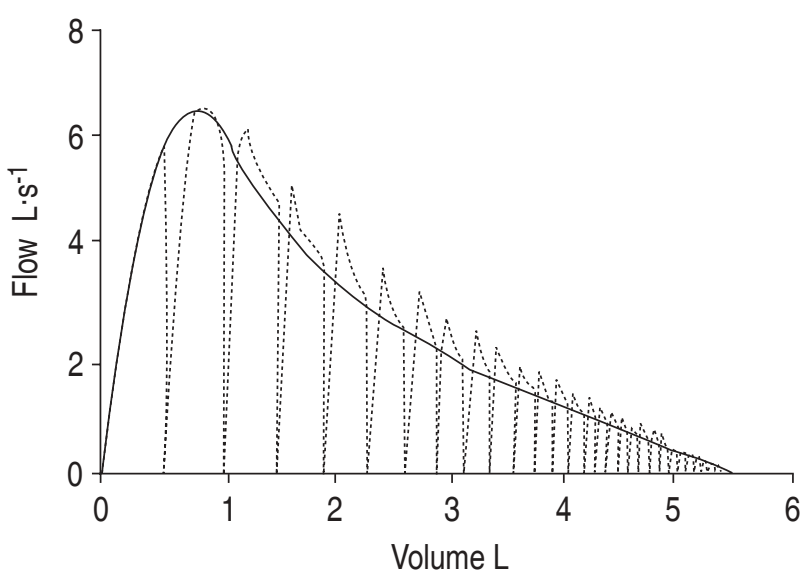

Fig. 7. - Form b curve. The flow after supramaximal flow remains higher than the flow observed in the basal curve.

pneumotachograph (PT), a rolling seal spirometer was chosen because the rolling seal has a better signalnoise relationship [4]. When a PT was used, the noise induced by valve work resulted in an SF overestimation as observed when performing interrupted MEFV curves with an explosive decompressor. Moreover, as one of the study objectives was accurate measurement of FEV1 in interrupted curves, the rolling-seal spirometer that primarily measures volume and then differentiates volume to give flow, seems to have better performance. The data from this study also demonstrated negligible SF when the expiratory manoeuver was performed with an explosive decompressor and measured with a rolling-seal spirometer (fig. 2).

By studying normal subjects, a significant inverse correlation between SF and flow limitation was observed. SF increases as forced mid-expiratory flow (FEF25\%-75\%) decreases [4]. It was also observed that the longer the closed-valve time the higher the SF. Neither observations can be explained by the theories suggested by KNUDSON et al. [2] and PEDERSEN et al. [3]. Therefore, the authors proposed that "pendelluft" might be another mechanism involved in the SF phenomenon. The authors also proposed that the increase in intrathoracic pressure during closed-valve time may result in gas explosive decompression when the valve opens, and so contributes to SF.

The first observation of this study is that the inverse correlation between SF and flow limitation that was observed in normal subjects [4], is also evident in asthmatic patients. The correlation between nonhomogeneity in lung emptying and SF is also supported by the results obtained in asthmatic patients when SF was measured after methacholine and salbutamol. SF decreased after salbutamol administration and increased by contrast with methacholine administration.

All the earlier mentioned SF mechanisms may be modified when salbutamol or methacholine are administered. Changes in SF after methacholine and salbutamol may be the result of changes in the volume of air contained in the flow-limiting segment (FLS), in airways configuration, in the compression degree of

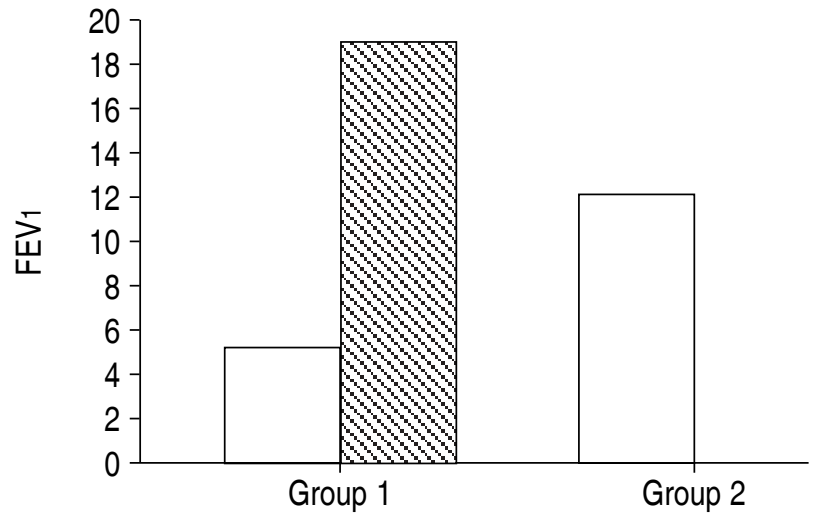

Fig. 8.-Prevalence of the two types of morphology according to forced expiratory volume in one second (FEV1). Group 1: FEV1 obtained from interrupted curve is higher than that obtained from basal curve. Group 2: FEV1 obtained from interrupted curve is lower than that obtained from basal curve. $\square$ : form a; $\mathbb{\mathbb { N }}$ : form $\mathrm{b}$.

the air contained in the thorax during flow interruptions, and in the heterogeneities in lung emptying. KNUDSON et al. [2] attributed SF to FLS emptying downstream to the choke point. Methacholine can increase the air volume in FLS and so it contributes to SF by upstream choke-point migration. Salbutamol can decrease SF by reducing volume in FLS by downstream choke-point migration. Pedersen et al. [3] measured air volume contained in the SF induced by $15 \mathrm{~Hz}$ interrupted curves and demonstrated that this volume was higher than that of the FLS. Therefore, Pederson et al. [3] suggested the implication of changes in the airways configuration in SF, from a transient unstable configuration that leads to the peak expiratory flow to a more stable configuration permitting less flow. The stable configuration persists until the next interruption. Methacholine and salbutamol can change the bronchial wall characteristics and so they help or hamper airways configuration change once the valve opens. This contribution, as far as the results from this study are concerned, is unknown. Methacholine that increases airway resistance and so modifies intrathoracic pressure, can increase SF. Salbutamol can produce the opposite effects. Finally, methacholine can increase and salbutamol can decrease nonhomogeneous lung emptying.

During valve open time, fast units contribute more than slow units to the expired flow. When the valve closes, slow units have higher volumes (and thus, higher elastic recoil pressure) than fast ones, so that during closure time there is airflow from slow to fast units. This could "refill" the empty fast units and increase its contribution to flow during the next valve open time, "pendelluft" participation in SF was also supported by another study with a lung mechanical model [9].

The analysis of interrupted expiratory curves morphology is complex. The different curve forms are related to changes in FEV1. Patients who increased FEV1 during interrupted curves in comparison with basal, have increased prevalence of form "b" curves. The characteristic of form "b" is that SF lasts longer after peak flow is reached until the next interruption. 
Methacholine administration induces forced expiratory flow fall because it increases low time constant units. The consequence of the larger range of time constants for different lung compartments is that there will be a large amount of "pendelluft" when the valve is closed and subsequently higher SF when the valve opens again.

The opposite situation may be observed following salbutamol administration in patients with increased flow limitation. Salbutamol decreases nonhomogeneity lung emptying with the subsequent lower difference in volume between alveolar units when valve closed time begins and lower SF immediately after valve reopening.

The observed form " $b$ " curve is defined by a persisting SF, after transient peak flow until the next interruption occurs. Thus, neither FLS emptying nor airway configuration changes could explain it. Fast units refilling during flow interruptions is therefore the main mechanism to explain form "b" curves. The association that the authors observed between FEV1 increase and form "b" curve prevalence implies that "pendelluft" is also a main contributor to the former. The fact that differences in flow between basal and interrupted curves are higher at high lung volumes also suggest nonhomogeneity of ventilation as demonstrated by WiLSON et al. [10].

This data support the idea that supramaximal flow is the result of several mechanisms. In asthmatic patients "pendelluft" involment in supramaximal increases, as flow limitation increases. The forced expiratory volume in one second behaviour, when interrupted forced expiratory curves were performed, requires further investigation.

\section{References}

1. Beardsmore CS, Wimmpress SP, Thomson AH. Maximum voluntary cough: an indication of airway function. Bull Eur Physiopathol Respir 1987; 23: 465472.

2. Knudson RJ, Mead J, Knudson DE. Contribution of airway collapse to supramaximal expiratory flows. J Appl Physiol 1974; 36: 653-667.

3. Pedersen OF, Lyager S, Ingram RH Jr. Airway dynamics in transition between peak and maximal expiratory flow. J Appl Physiol 1985; 59: 1733-1745.

4. Sala H, Galindez F, Badolati A, Rodenstein D. Relationship between supramaximal flows and flow-limiting mechanisms. Eur Respir J 1996; 9: 512516.

5. ATS. Standarization of spirometry. Am Rev Respir Dis 1987; 136: 1285-1298.

6. Chathman M, Bleecker ER, Norman P, et al. A screening test for airways reactivity. Chest 1982; 82: $15-18$.

7. Gardner RM, Hankinson JL, West BJ. Evaluating comercially available spirometers. Am Rev Respir Dis 1980; 121: 73-82.

8. Petisevsky ML, Lyons LD, Smith AA, Epler GR, Gaensler EA. Calibration of time derivates of forced vital capacity by explosive decompression. Am Rev Respir Dis 1980; 121: 343-350.

9. Sala H, Veritier C, Rodenstein D, Alzaibar C, Llistro G. Can "pendelluft" explain supramaximal flows during interrupted forced expirations. Eur Respir $J$ Suppl 1999; 14: Suppl. 30, 393.

10. Wilson TA, Fredberg JJ, Rodarte JR. Interdependence of regional expiratory flow. J Appl Physiol 1985; 59: 1924-1928. 